\title{
Polyamine enhances the regeneration of reduced glutathione by the activation of NADP-dependent dehydrogenases in yeast
}

\author{
Yasuko Tanemura, Keiko Murakami, Miyako Haneda and Masataka Yoshino \\ Department of Biochemistry, Aichi Medical University School of Medicine, Nagakute, Aichi 480-1195, Japan \\ (Received 13 January 2004; and accepted 16 February 2004)
}

\begin{abstract}
Effect of polyamine on the regeneration of reduced glutathione (GSH) was analyzed in yeast. Spermine stimulated the reduction of oxidized glutathione in the presence of glucose 6-phosphate or isocitrate in permeabilized yeast cells. Stimulation of GSH formation was due to the enhancement of NADPH (reduced nicotinamide adenine dinucleotide phosphate) supply. Polyamine activated mitochondrial NADP (nicotinamide adenine dinucleotide phosphate)-isocitrate dehydrogenase (EC 1.1.1.42) and cytosolic glucose 6-phosphate dehydrogenase (EC 1.1.1.49) from yeast. Activating action of polyamine was largely the effect on the maximal velocity of these enzymes, and the order of effectiveness as the activators was spermine $>$ spermidine $>$ putrescine. The concentrations of spermine required for $50 \%$ activation of the enzymes were about $1-2 \mathrm{mM}$, values that were within the range of physiological concentrations of spermine. Polyamine can act as an antioxidant by stimulating the regeneration of reduced glutathione, the principal reactive oxygen scavenger, through the activation of cytosolic glucose 6-phosphate dehydrogenase and mitochondrial NADPisocitrate dehydrogenase as the NADPH-generating enzymes in yeast.
\end{abstract}

Polyamines, which accumulate with cellular proliferation, are responsible for anabolic processes in vivo $(1,16)$. Yeast cells are able to grow both aerobically and anaerobically. Under aerobic conditions, cells are exposed to various reactive oxygen species resulting from normal aerobic metabolism, and spermine and spermidine, the representatives of polyamine, are essential for aerobic growth of yeast $(1,3)$. These results suggest that polyamines may act as antioxidants by protecting cell components from oxidative damages $(4,6)$. Antioxidant action of polyamine has been explained by preventing the generation of reactive oxygen species $(12,17)$, and by scavenging free radical (9-11). Of free radical scavengers, glutathione plays a principal role in the antioxidant system, and oxidized glutathione formed by the reaction with oxygen radicals should be regener-

Correspondence to: Dr. Masataka Yoshino

Tel: +81-52-264-4811(ext. 2360), Fax: +81-561-61-4056

E-mail: yoshino@aichi-med-u.ac.jp ated to reduced form by the action of glutathione reductase (EC 1.6.4.2), which requires reduced NADP coenzymes. Thus, supply of NADPH is one of the limiting factors of antioxidant action. Here we report the stimulation by polyamine of the regeneration of glutathione under the in situ conditions of yeast cells, and further analyzed the activating effect of polyamine on the NADPH-generating enzymes. Enhanced formation of reduced glutathione by polyamine can be explained by the activation of NADP-isocitrate dehydrogenase (EC 1.1.1.42) and glucose 6-phosphate dehydrogenase (EC 1.1.1.49), the principal NADPH-generating enzymes. Polyamine can play a central role in antioxidant defense system.

\section{MATERIALS AND METHODS}

Materials. The sources of materials used in this work as follows: threo-Ds-isocitrate, NADP, glucose 6-phosphate, 2-oxoglutarate, spermine, spermid- 
ine, and putrescine from Sigma-Aldrich-Japan (Tokyo, Japan), 3-( $N$-morpholino) propanesulfonic acid (MOPS) from Dojindo Co. (Kumamoto, Japan), and the purified yeast enzymes including recombinant mitochondrial NADP-isocitrate dehydrogenase $(17.7 \mu \mathrm{mol} / \mathrm{min}$ per mg protein), NAD-isocitrate dehydrogenase (EC 1.1.1.41) $(31 \mu \mathrm{mol} / \mathrm{min}$ per $\mathrm{mg}$ protein) and glucose 6-phosphate dehydrogenase ( $292 \mu \mathrm{mol} / \mathrm{min}$ per $\mathrm{mg}$ protein) from Oriental Yeast Co. (Tokyo, Japan).

Determination of enzyme activity. Enzyme activity was measured by following the change in absorbance at $340 \mathrm{~nm}$ at $37^{\circ} \mathrm{C}$. Reaction mixture of $1 \mathrm{ml}$ for determining NADP-isocitrate dehydrogenase activity contained $100 \mathrm{mM}$ MOPS-KOH buffer $(\mathrm{pH}$ 6.85 ), $0.5 \mathrm{mM} \mathrm{MgCl}_{2}$, various concentrations of NADP, threo-Ds-isocitrate and polyamine, and the purified enzyme (14). The assay medium of $1 \mathrm{ml}$ for the determination of glucose 6-phosphate dehydrogenase contained $100 \mathrm{mM}$ MOPS-KOH buffer $(\mathrm{pH}$ 6.85 ), the enzyme, and various concentrations of glucose 6-phosphate and NADP in the absence and presence of polyamine. Reaction was initiated by addition of each enzyme. Kinetic parameters were expressed as mean \pm SD for 5 independent experiments using the nonlinear regression analysis (5).

For the estimation of cellular enzyme activity levels, an in situ assay was used with the permeabilized yeast cells as the enzyme (15).

Formation of GSH (reduced glutathione) in permeabilized yeast. Saccharomyces cerevisiae was cultivated in liquid culture containing rich medium (1\% yeast extract, $2 \%$ bacto-peptone and $2 \%$ glucose). Yeast was grown aerobically at $30^{\circ} \mathrm{C}$ for $24 \mathrm{~h}$, and collected cells were permeabilized by treatment with toluene (15). The reaction mixture for the formation of reduced glutathione contained $0.5 \mathrm{mM}$ NADP, 1.7 $\mathrm{mM}$ oxidized glutathione, $40 \mathrm{mM}$ Tris- $\mathrm{HCl}$ buffer (pH 7.1), $0.5 \mathrm{mM}$ glucose 6-phosphate or $0.4 \mathrm{mM}$ threo-Ds-isocitrate, permeabilized yeast cells (1

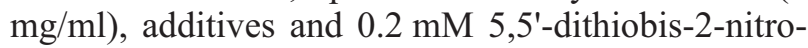
benzoic acid (DTNB) in a total volume of $1 \mathrm{ml}$. Formation of reduced glutathione was followed by the increase in the absorbance at $412 \mathrm{~nm}$.

\section{RESULTS}

Reduced glutathione plays a principal role in cellular antioxidant system: GSH can scavenge reactive oxygen species directly and further with glutathione peroxidase (EC 1.11.1.9) reaction. Oxidized glu- tathione formed is regenerated to a reduced form by glutathione reductase (EC 1.6.4.2). We determined the cellular enzyme activities on the NADPH-generating reactions in yeast cells grown on the medium with glucose as carbon source. Activities of NADPisocitrate dehydrogenase and glucose 6-phosphate dehydrogenase were 9.1 and $19.6 \mu \mathrm{mol} / \mathrm{min}$ per $\mathrm{g}$ yeast, respectively. However, malic enzyme (EC 1.1.1.40), an NADPH-generating enzyme in mammalian tissues (2) showed negligible activity in yeast.

Effect of polyamines on the regeneration of reduced glutathione was analyzed by using permeabilized yeast cells. Reduced glutathione was not at all formed from oxidized glutathione without glucose 6phosphate, and spermine itself could not stimulate the reduction of glutathione (Fig. 1A). Addition of glucose 6-phosphate enhanced the reduction of oxidized glutathione, and further addition of spermine markedly stimulated the formation of reduced glutathione (Fig. 1A). When isocitrate was substituted for glucose 6-phosphate as the substrate for the NADPH-generating enzyme, oxidized glutathione could be negligibly reduced without metal or spermine. However, addition of spermine or $\mathrm{Mg}^{2+}$ ion effectively enhanced the formation of reduced glutathione. Addition of spermine plus $\mathrm{Mg}^{2+}$ further stimulated the reduction of glutathione (Fig. 1B). These results suggest that polyamine enhances the supply of NADPH due to the activation of NADPisocitrate dehydrogenase and glucose 6-phosphate dehydrogenase, resulting in the stimulation of glutathione formation.

We further examined the effects of polyamines on the NADP-isocitrate dehydrogenase purified from yeast. The $\mathrm{Km}$ and Vmax values of the enzyme were calculated to be $4.29 \pm 1.4 \mu \mathrm{M}$ and $4.17 \pm 0.12$ $\mu \mathrm{mol} / \mathrm{min}$ per $\mathrm{mg}$ protein, respectively. All the polyamines tested increased the maximal velocity of the enzyme. The order of effectiveness of the polyamine as an activator was spermine $>$ spermidine $>$ putrescine. Spermine effectively increased the maximal velocity to $14.8 \pm 0.6 \mu \mathrm{mol} / \mathrm{min}$ per $\mathrm{mg}$ protein, but did not affect the $\mathrm{Km}$ value for isocitrate and NADP as the substrates (Fig. 2). Spermidine and putrescine also increased the maximal velocity without affecting $\mathrm{Km}$ values for isocitrate and NADP (Fig. $2 \mathrm{C}$ and $2 \mathrm{D}$ ).

Effect of increasing concentrations of polyamines on the NADP-isocitrate dehydrogenase activity was shown in Fig. 3. The concentration necessary for $50 \%$ activation of this enzyme activity, $\mathrm{A}_{0.5}$ value for spermine was about $2-3 \mathrm{mM}$ under the condi- 

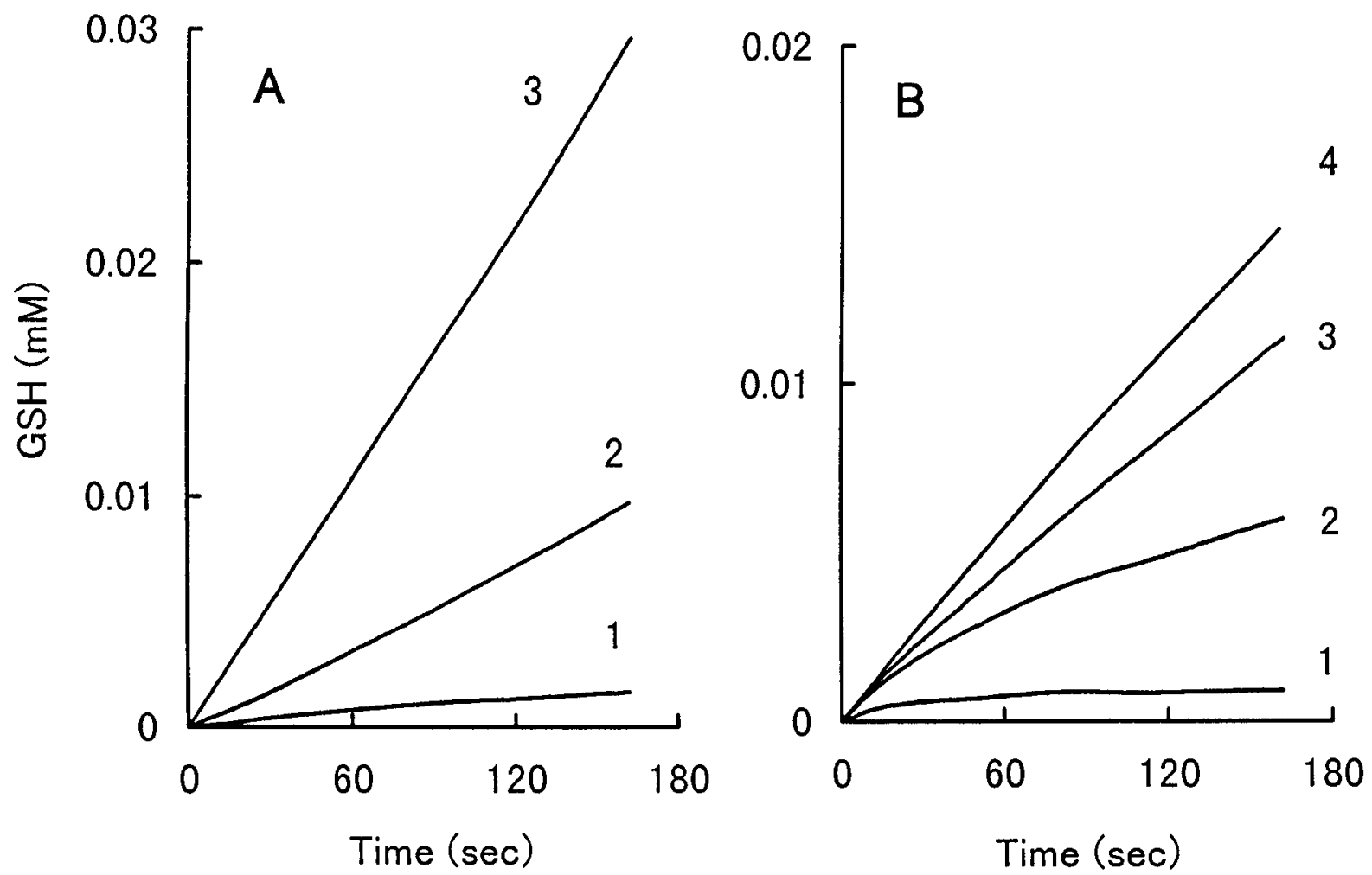

Fig. 1 Effect of spermine on the formation of reduced glutathione. Permeabilized yeast cells (1 mg/ml) were incubated at 37 ${ }^{\circ} \mathrm{C}$ in the reaction mixture containing $0.5 \mathrm{mM}$ NADP, $1.7 \mathrm{mM}$ oxidized glutathione, $40 \mathrm{mM}$ Tris- $\mathrm{HCl}(\mathrm{pH} 7.1), 0.2 \mathrm{mM} \mathrm{5,5'-}$ dithiobis-2-nitrobenzoic acid and following additives in a total volume of $1 \mathrm{ml}$. Formation of reduced glutathione was followed by the increase in the absorbance at $412 \mathrm{~nm}$. (A) Glucose 6-phosphate dehydrogenase as an NADPH-generating system. Curve 1, $0.4 \mathrm{mM}$ spermine added. Curve 2, $0.5 \mathrm{mM}$ glucose 6 -phosphate added. Curve 3, $0.4 \mathrm{mM}$ spermine plus $0.5 \mathrm{mM}$ glucose 6-phosphate. (B) NADP-Isocitrate dehydrogenase as an NADPH-generating system. Reaction mixture contained $0.4 \mathrm{mM}$ threo-Ds-isocitrate. Curve 1, no addition. Curve 2, $0.4 \mathrm{mM}$ spermine added. Curve $3,5 \mathrm{mM} \mathrm{MgCl}_{2}$ added. Curve 4, $5 \mathrm{mM}$ $\mathrm{MgCl}_{2}$ plus $0.4 \mathrm{mM}$ spermine added.

tions where the substrate concentrations were kept at 20 and $100 \mu \mathrm{M}$ (Fig. 3A). This $\mathrm{A}_{0.5}$ values are within the physiological concentration range in cells (16). Spermidine and putrescine showed less activating effect on the enzyme (Fig. 3B). On the other hand, NAD-isocitrate dehydrogenase from yeast was not at all activated, but rather inhibited (data not shown), indicating that polyamine effect may be specific for the NADP-isocitrate dehydrogenase.

Spermine also activated the yeast glucose 6-phosphate dehydrogenase (Fig. 4): the activation was largely on the maximal velocity with a little change in the affinity for the substrate NADP (Fig. 4C and 4D). Effect of increasing concentrations of polyamine on the glucose 6-phosphate dehydrogenase activity was shown in Fig. 3B. The effectiveness of polyamine as an activator of glucose 6-phosphate dehydrogenase was similar to the effect on NADPisocitrate dehydrogenase.

\section{DISCUSSION}

Oxygen can cause serious stress to living organism by producing reactive oxygen species, such as superoxide anion, hydrogen peroxide and hydroxyl radical. In response to the oxidative stress, most organisms have developed various defense mechanisms by the expression of antioxidant enzymes, and by detoxifying oxygen radicals with various antioxidants. A number of reports have highlighted the key role of glutathione in the response to oxidative stress of cells (7): reduced glutathione can scavenge reactive oxygen directly and further by the action of glutathione peroxidase. Glutathione, thus, contributes to the protection of tissues from the injury by reactive oxygen species in the aerobic growing yeast (13). Oxidized glutahione formed by scavenging reactive oxygen species should be regenerated to the reduced form by the action of glutathione reductase that utilizes NADPH as the 



Fig. 2 Effect of the concentrations of threo-Ds-isocitrate (A, B) and NADP (C, D) on the activity of yeast NADP-isocitrate dehydrogenase in the absence and presence of polyamine. Reaction mixture of $1 \mathrm{ml}$ contained $100 \mathrm{mM} \mathrm{MOPS-KOH}$ buffer (pH 6.85), $0.5 \mathrm{mM} \mathrm{MgCl}_{2}$, various concentrations of threo-Ds-isocitrate or NADP, and the enzyme in the absence (O) and presence of $2 \mathrm{mM}$ spermine $(\bigcirc)$, spermidine $(\Delta)$ or putrescine $(\square)$. (A) threo-Ds-isocitrate saturation curves in the absence and presence of various polyamine. NADP concentration was kept at $0.1 \mathrm{mM}$. (B) Double reciprocal plot of the data A. (C) NADPsaturation curves. threo-Ds-isocitrate was kept at $0.1 \mathrm{mM}$. (D) Double reciprocal plot of the data C. Points represent experimental data, and lines are theoretically drawn according to the non-linear regression analysis (5). Values of Km and Vmax are calculated as follows. Km for threo-Ds-isocitrate, $4.29 \pm 1.4 \mu \mathrm{M}$; Km for NADP, $8.28 \pm 1.57 \mu \mathrm{M}$; Vmax, $4.17 \pm 0.12$ (none) and $14.8 \pm 0.6 \mu \mathrm{mol} / \mathrm{min}$ per $\mathrm{mg}$ protein (spermine added).
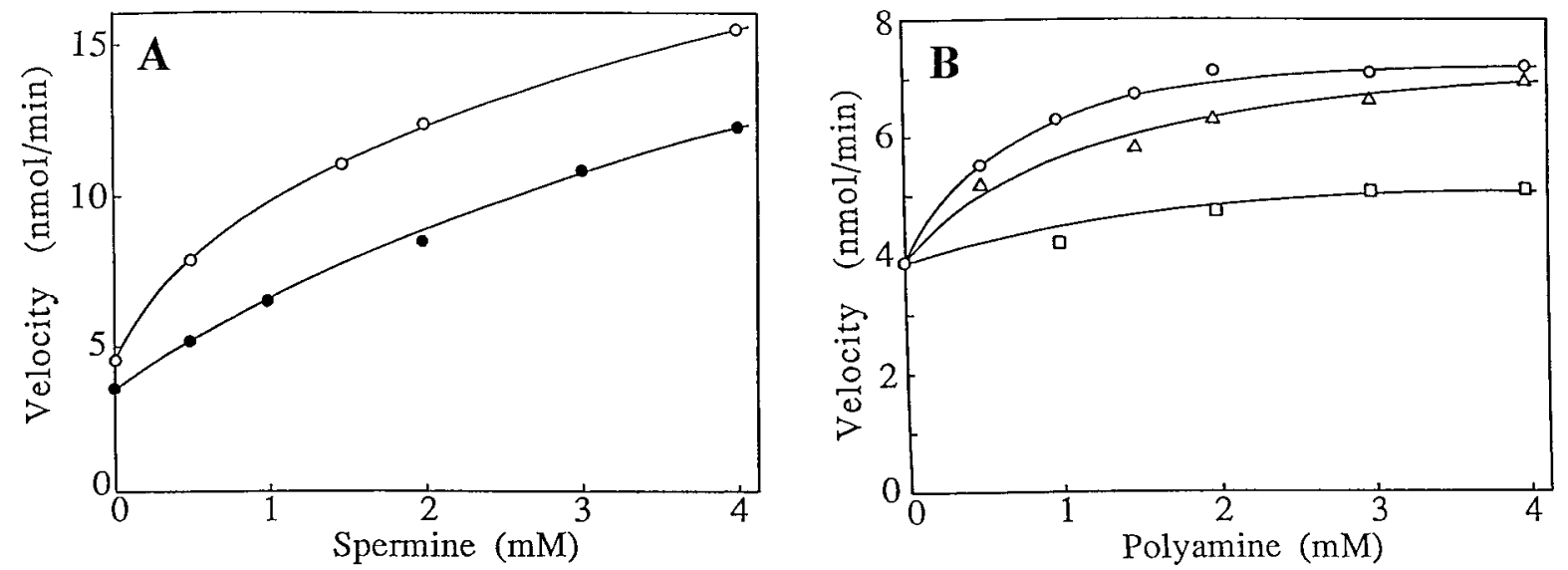

Fig. 3 Effect of polyamine on the activity of mitochondrial NADP-isocitrate dehydrogenase and glucose 6-phosphate dehydrogenase. (A) Effect of spermine on the NADP-isocitrate dehydrogenase in the presence of different concentrations of threo-Ds-isocitrate. Reaction mixture was similar to that of the legend to Fig. 1 except that spermine concentration was varied in the presence of $20 \mu \mathrm{M}(\mathrm{O})$ and $100 \mu \mathrm{M}(\mathrm{O})$ threo-Ds-isocitrate. (B) Effect of polyamine on the activity of glucose 6-phosphate dehydrogenase. Reaction mixture was similar to that of the legend to Fig. 2 except that polyamine concentration was varied in the presence of $0.1 \mathrm{mM}$ NADP and glucose 6 -phosphate. $O$, spermine; $\Delta$, spermidine; $\square$, putrescine. 

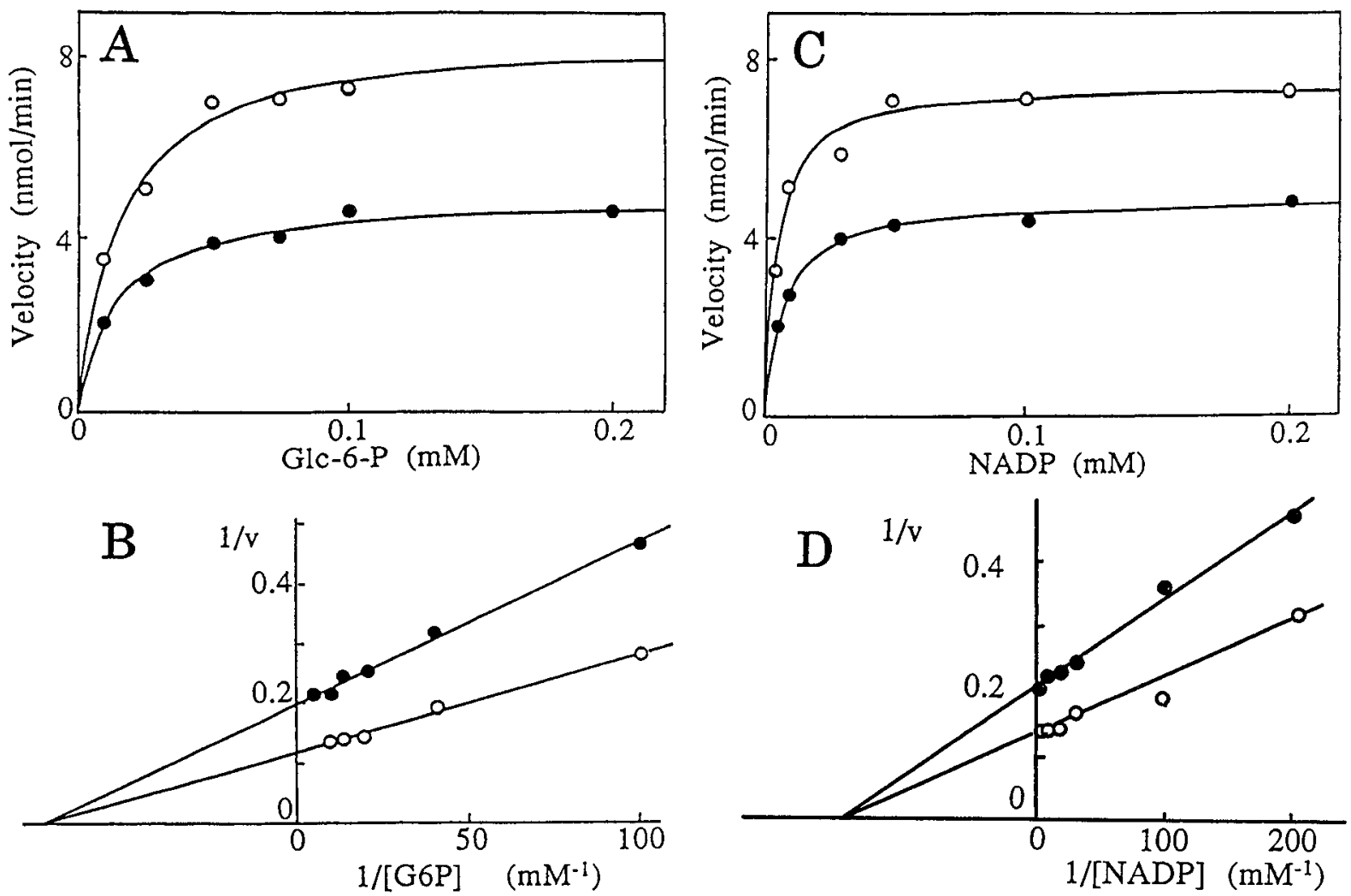

Fig. 4 Effect of the concentrations of glucose 6-phosphate (A, B) and NADP (C, D) on the activity of yeast glucose 6-phosphate dehydrogenase in the absence and presence of polyamine. Reaction mixture of $1 \mathrm{ml}$ contained $100 \mathrm{mM} \mathrm{MOPS}-\mathrm{KOH}$ buffer ( $\mathrm{pH}$ 6.85), various concentrations of glucose 6-phosphate or NADP in the absence (O) and presence of $2 \mathrm{mM}$ spermine (O). (A) Glucose 6-phosphate saturation curves. NADP concentration was kept at $0.2 \mathrm{mM}$. (B) Double reciprocal plot of the data A. (C) NADP-saturation curves. Glucose 6-phosphate was kept at $0.2 \mathrm{mM}$. (D) Double reciprocal plot of the data C. Points represent experimental data, and lines are theoretically drawn according to the non-linear regression analysis (5). Values of $\mathrm{Km}$ and $\mathrm{Vmax}$ are calculated as follows. $\mathrm{Km}$ for glucose 6-phosphate, $13.4 \pm 1.1 \mu \mathrm{M}$; $\mathrm{Km}$ for NADP, $6.93 \pm 0.33 \mu \mathrm{M}$; Vmax, $4.91 \pm 0.13$ (none) and $8.46 \pm 0.33 \mu \mathrm{mol} / \mathrm{min}$ per $\mathrm{mg}$ protein (spermine added).

coenzyme. Production of NADPH is, thus, considered to act as a key step in GSH regeneration. NADPH-generating system consists of glucose 6phosphate dehydrogenase, NADP-isocitrate dehydrogenase and malic enzyme. Under the experimental conditions of yeast cells that lacks malic enzyme, regeneration of NADPH depends on NADP-linked isocitrate dehydrogenase and glucose 6-phosphate dehydrogenase (8). NADP-isocitrate dehydrogenase consists of two isozymes: mitochondrial and cytosolic forms. Mitochondrial NADP-isocitrate dehydrogenase is expressed at high levels irrespective of carbon sources, but cytosolic NADP-isocitrate dehdyrogenase is expressed in yeast grown on nonfermentable carbon sources (18). The mitochondrial NADP-isocitrtate dehydrogenase, thus, acts as the only enzyme regenerating reduced NADP in mitochondria of yeast grown on glucose. Glucose 6-phosphate dehydrogenase is localized in cytosol, and plays a principal role in the NADPH supply in cytosol.

The present study showed that polyamines, in particular spermine and spermidine, stimulate the regeneration of glutathione by activating NADP-isocitrate dehydrogenase and glucose 6-phosphate dehydrogenase, suggesting that polyamine can act as an antioxidant by stimulation of the glutathione regeneration. A vast amount of literature has indicated the anabolic role of polyamines related to increased cellular proliferation in animal and microbial cells. Polyamine accumulation is accompanied by an increase in the rate of nucleic acid synthesis as well as protein synthesis: many enzymes of DNA, RNA and protein biosynthesis are activated by polyamines (1). Several lines of evidence showed the function of polyamine as an antioxidant under the in vitro and in vivo conditions. Yeast cells require spermine or spermidine for aerobic growth, 
and polyamine is presumed to protect cell components from damage resulting from oxidation (3). Antioxidant effect of polyamine has been considered to be due to the action preventing the generation of reactive oxygen species by the chelation of metals as prooxidants $(12,17)$, and also scavenging reactive oxygen species $(10,11)$. Spermine as a free radical scavenger was shown to protect DNA from oxidative damage (9). In addition to these known antioxidant characteristics, polyamine-dependent stimulation of glutathione regeneration demonstrated in this paper presents a new insight on the antioxidant properties of this compound.

Activating effect of polyamine on the NADPHgenerating enzymes was specific, and was largely on the maximal velocity. The concentrations required for $50 \%$ activation, the $\mathrm{A}_{0.5}$ values for spermine were within the intracellular concentration range $(1,16)$, indicating that polyamine can act as a physiological effectors of these enzymes. In conclusion, activation of NADPH-generating enzymes by polyamine is responsible for the regeneration of glutathione in cytosol and mitochondria. Antioxidant nature of polyamine should be evaluated by the enhanced supply of NADPH contributing to increased regeneration of reduced glutathione in addition to the known properties scavenging reactive oxygen species.

\section{REFERENCES}

1. Abrahams AK and Pihl A (1981) Role of polyamines in macromolecular synthesis. Trends Biochem Sci 6, 106-107.

2. Andres A, Satrustegui J and Machado A (1980) Development of NADPH-producing pathways in rat heart. Biochem $J \mathbf{1 8 6}$, 799-803.

3. Balasundaram D, Tabor CW and Tabor H (1991) Spermidine or spermine is essential for the aerobic growth of Saccharomyces cerevisiae. Proc Natl Acad Sci USA 88, 5872-5876.

4. Balasundaram D, Tabor CW and Tabor H (1993) Oxygen toxicity in a polyamine-depleted spe 2 delta mutant of Saccharo- myces cerevisiae. Proc Natl Acad Sci USA 90, 4693-4697.

5. Duggleby RG (1981) A nonlinear regression program for small computers. Anal Biochem 110, 9-18.

6. Farbiszewski R, Bielawska M, Szymanska M and Skrzydlewska E (1996) Spermine partially normalizes in vivo antioxidant defense potential in certain brain regions in transiently hypoperfused rat brain. Neurochem Res 21, 1497-1503.

7. Grant CM (2001) Role of glutathione/glutarexoxin and thioredoxin systems in yeast growth and response to stress conditions. Mol Microbiol 39, 533-541.

8. Haselbeck RJ and McAlister-Henn L (1993) Function and expression of yeast mitochondrial NAD- and NADP-specific isocitrate dehydrogenase. $J$ Biol Chem 268, 12116-12122.

9. Ha HC, Sirisoma NS, Kuppusamy P, Zweier JL, Woster PM and Casero RA Jr (1998) The natural polyamine spermine functions directly as a free radical scavenger. Proc Natl Acad Sci USA 95, 11149-11145.

10. Khan AU, Mei YH and Wilson T (1992) A proposed function for spermine and spermidine: Protection of replicating DNA against damage by singlet oxygen. Proc Natl Acad Sci USA 89, 11426-11427.

11. Khan AU, Di Mascio P, Medeiros $M H G$ and Wilson $T$ (1992) Spermine and spermidine protection of plasmid DNA against single-strand breaks induced by singlet oxygen. Proc Natl Acad Sci USA 89, 11428-11430.

12. Lovaas E (1994) Hypothesis: Spermine may be an important epidermal anti-oxidant. Med Hypothesis 45, 59-67.

13. Muller EG (1996) A glutathione reductase mutant of yeast accumulates high levels of oxidized glutathione and requires thioredoxin for growth. Mol Biol Cell 7, 1805-1813.

14. Murakami K, Iwata S, Haneda M and Yoshino M (1997) Role of metal cations in the regulation of NADP-linked isocitrate dehydrogenase from porcine heart. BioMetals 10, 169-174.

15. Murakami K, Nagura H and Yoshino M (1980) Permeabilization of yeast cells: Application to study on the regulation of AMP deaminase activity in situ. Anal Biochem 105, 407-413.

16. Tabor CW and Tabor H (1985) Polyamines in microorganisms. Microbiol Rev 49, 81-99.

17. Tadolini B (1988) Polyamine inhibition of lipoperoxidation. The influence of polyamines on iron oxidation in the presence of compounds mimicking phospholipid polar heads. Biochem $J$ 249, 33-36.

18. Zhao W-N and McAlister-Henn L (1996) Expression and gene disruption analysis of the isocitrate dehydrogenase family in yeast. Biochemistry 35, 7873-7878. 